(2)

\title{
Bone mineral density is increased after a 16-week resistance training intervention in elderly women with decreased muscle strength
}

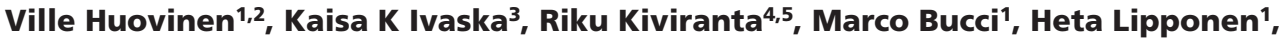 \\ Samuel Sandboge ${ }^{6,7}$, Juho Raiko', Johan G Eriksson ${ }^{6,7,8}$, Riitta Parkkola ${ }^{1,2}$, \\ Patricia lozzo ${ }^{1,9}$ and Pirjo Nuutila ${ }^{1,4}$ \\ ${ }^{1}$ Turku PET Centre, University of Turku, Turku, Finland, ${ }^{2}$ Department of Radiology, University of Turku and \\ Turku University Hospital, Turku, Finland, ${ }^{3}$ Department of Cell Biology and Anatomy, Institute of Biomedicine, \\ University of Turku, Turku, Finland, ${ }^{4}$ Department of Endocrinology, Turku University Hospital, Turku, Finland, \\ ${ }^{5}$ Departments of Medicine and Medical Biochemistry and Genetics, University of Turku, Turku, Finland, \\ ${ }^{6}$ Folkhälsan Research Centre, Helsinki, Finland, ${ }^{7}$ Department of Chronic Disease Prevention, National Institute for \\ Health and Welfare, Helsinki, Finland, ${ }^{8}$ Department of General Practice and Primary Health Care, University of \\ Helsinki and Helsinki University Hospital, Helsinki, Finland, and ${ }^{9}$ Institute of Clinical Physiology, National Research \\ Council (CNR), Pisa, Italy
}

\author{
Correspondence \\ should be addressed \\ to P Nuutila \\ Email \\ pirnuu@utu.fi
}

\begin{abstract}
Objective: Non-pharmacological interventions are important in reducing risk for osteoporotic fractures. We investigated the effects of a 16-week individualized resistance training intervention on bone mineral density (BMD), bone turnover markers and 10-year relative risk (RR) for osteoporotic fracture.

Design: Interventional study with a follow-up.

Methods: In total, 37 elderly women (mean age $71.9 \pm 3.1$ years) with decreased muscle strength participated in the resistance training intervention three times per week with $60 \mathrm{~min}$ per session for 16 weeks under the supervision of a licensed physiotherapist. Total hip BMD with quantitative CT, bone markers (sclerostin, osteocalcin, CTX, PINP, IGF-1, 25(OH)-D) and 10-year RR for osteoporotic fracture were measured at baseline, post-intervention and at 1-year follow-up after the end of the intervention. Eleven age- and sex-matched controls did not participate in the intervention but were studied at baseline and at 1-year follow-up.

Results: Resistance training seemed to increase total hip BMD by $6 \%(P=0.005)$. Sclerostin $(P<0.001)$ and total osteocalcin $(P=0.04)$ increased while other bone markers remained unchanged. A 10-year RR for major osteoporotic and hip fracture remained unchanged. At follow-up total hip BMD $(P<0.001)$ decreased back to the baseline level with a simultaneous decrease in serum sclerostin $(P=0.045), C T X(P<0.001)$ and an increase in $25(\mathrm{OH})-\mathrm{D}(P<0.001)$, 10-year RR for major osteoporotic $(P=0.002)$ and hip fracture $(P=0.01)$.

Conclusions: Our findings suggest an important role of continuous supervised resistance training for the prevention of osteoporotic fractures in elderly women with decreased muscle strength.

\section{Introduction}

Low bone mineral density (BMD) is a major risk factor for osteoporotic fracture (1). In addition to pharmacological interventions (2), resistance training is an effective
() 2016 European Society of Endocrinology Printed in Great Britain method to maintain $(3,4)$ or even increase BMD and muscle strength (5-12), which together may reduce the risk of fracture through increased balance (13) or an 
osteogenic effect stimulated by mechanical loading (14). Osteocytes are the mechanosensors in bone tissue (15) that coordinate the osteogenic response to mechanical loading at least in part through the expression of sclerostin (16), which is an osteocyte-secreted soluble antagonist of the Wnt/ $\beta$-catenin signaling pathway and regulates osteoblast differentiation and activity and is a negative regulator of bone mass (17). However, paradoxically in a few studies, sclerostin has been found to positively associate with BMD in postmenopausal women (18-20).

The WHO Fracture Risk Assessment Tool (FRAX) integrates many risk factors for osteoporotic fractures including hip BMD and can be used to estimate individual 10-year relative risk (RR) for osteoporotic fracture (21). The gold standard method in clinical use for measuring BMD is dual x-ray absorptiometry (DXA) (22). QCT is a technique used to measure hip BMD with computed tomography (CT) with a calibration phantom to convert Hounsfield units (HU) to BMD values (23). BMD results between hip QCT (CTXA hip) and conventional DXA have a highly positive correlation $(24,25)$. However, it is not known whether a resistance training intervention results in a long-term improvement of hip BMD as measured with QCT in women with decreased muscle strength.

The aim of this study was to investigate the effects of a 16-week supervised resistance training intervention on hip BMD, bone markers and 10-year RR for osteoporotic fracture in elderly women with decreased muscle strength. Furthermore, our aim was to investigate whether the possible favorable outcomes are sustained 1 year after the end of the intervention. We hypothesized that resistance training intervention results in an increase in hip BMD and is accompanied with changes in bone markers and, consequently, a decrease in 10-year RR for osteoporotic fracture. We further hypothesized that the putative increase in BMD is not sustained after cessation of the resistance training intervention. To address these questions, we used the QCT imaging method to assess hip BMD, blood sampling and FRAX (http://www.shef. ac.uk/FRAX/) before and after the resistance training intervention and 1 year after the end of the intervention.

\section{Subjects and methods}

\section{Study population}

Study subjects were recruited at the Folkhälsan Research Center, Helsinki, Finland, between May 21, 2012, and December 4, 2013, from the Helsinki Birth Cohort Study (HBCS) II (26), which included 13345 subjects born during
1934-1944. The eligible study subjects were selected from a sub-cohort of 2003 subjects that had been deeply clinically characterized throughout the years. 37 subjects with decreased handgrip strength values and 11 age- and sex-matched controls with normal handgrip strength values were successfully recruited for the study. Data for the handgrip strength measurement was obtained from the earlier conducted clinical study (27). Inclusion criteria for the subjects with decreased handgrip strength were a handgrip strength value below the median value of all HBCS participants assessed between 2001 and 2004, age between 68 and 78 years, female gender and none of the exclusion criteria present. Inclusion criteria for the controls were handgrip strength value above the median value of all HBCS participants assessed between 2001 and 2004, age between 68 and 78 years, female gender and none of the exclusion criteria present. The exclusion criteria for all subjects included diabetes requiring insulin treatment or fasting glucose above $7 \mathrm{mmol} / \mathrm{L}$ at the last visit before the enrollment. Subjects currently smoking or with comorbidities influencing insulin sensitivity or with contraindications for participating in an exercise intervention (e.g., chronic atrial fibrillation and pacemaker) or an MRI study were also excluded. Before and after the intervention until the follow-up visit, none of the subjects used intraoral glucocorticoids or medications other than calcium and vitamin D, which affects calcium or bone metabolism. None of the subjects had suffered from previous hip fracture. No differences were found in age $(P=0.22)$ or BMI $(P=0.56)$ between included and excluded women subjects. Sample size was based on our previous study investigating the effect of resistance training on thigh muscle and whole-body insulin sensitivity (28). A sample size of 37 subjects with decreased muscle strength would allow us to detect a $4.9 \%$ change in hip BMD at a significance level of $95 \%$ and with $80 \%$ power. The ethics committee of the Hospital District of Southwestern Finland approved the studies (26/180/2012), which were conducted according to the principles of the Declaration of Helsinki. All subjects gave their written informed consent before studies. The study is registered to Clinicaltrials.gov (NCT01931540).

\section{Study design}

The study design is depicted in Fig. 1. Intervention and control groups were studied at baseline, and only the intervention group was studied after a 16-week supervised resistance training intervention. A subset of 19 intervention subjects and 6 control subjects were studied 1 year after the end of the intervention. The average time between the pre-intervention and post-intervention visit 


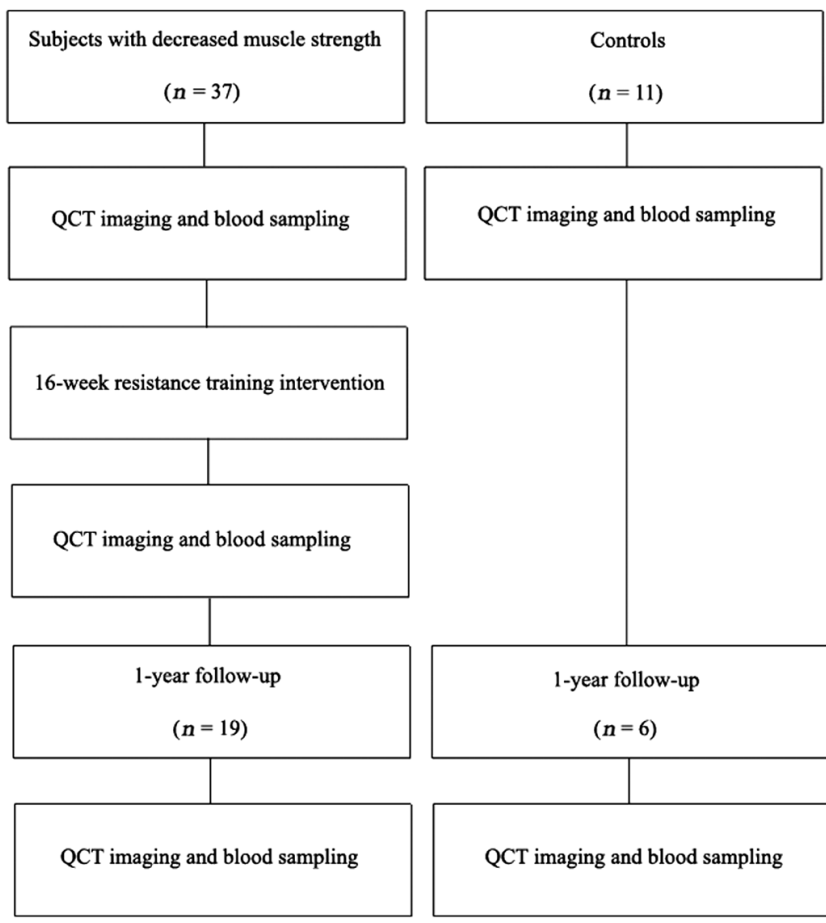

\section{Figure 1}

Study design.

was $5.1 \pm 0.7$ months, between post-intervention and follow-up visit was $12.3 \pm 1.5$ months and between preintervention and follow-up visit was $17.8 \pm 3.1$ months.

After the first study visit, the intervention group underwent an individualized resistance training intervention. The intervention consisted of three sessions per week, which lasted 60 min per session and was under the supervision of a trained licensed physiotherapist for 16 weeks. Resistance training sessions started with warm-up (10 min) with cycle and/or elliptical ergometer. They continued with different resistance exercises targeting large muscle groups of the lower and upper body (e.g., leg presses, chest presses, seated rows, abdominal crunches, back extensions, seated leg curls and hip abductions). At each station, subjects completed three sets of 8-15 repetitions with a load that corresponded to $50-80 \%$ of estimated one repetition maximum (RM). One RM for each exercise was estimated from 8-RM tests by applying Epley's formula (29). Progress in muscle strength was measured once a month and the loads for the following month were adjusted as appropriate. Adherence (actualized individual exercise frequency per week divided by maximum exercise amount per week) to exercise was $78.6 \pm 10.8 \%$. All the subjects who were not prescribed with anti-osteoporotic treatment (other than calcium or vitamin D) after intervention (32 from the intervention group and 11 controls) were invited to a follow-up visit that took place 1 year after the end of the intervention. About 25 subjects (19 from the intervention group and 6 controls) participated in this visit. There were no differences in the baseline characteristics among the subjects that were included in or excluded from the follow-up except for serum sclerostin, which was lower in the included group ( $P=0.004$ ) (Table 1$)$ even after adjusting for muscle strength. This difference found was no longer present at the post-intervention visit $(P=0.31)$. No difference in the exercise effect on serum sclerostin was observed between the included and excluded subjects $(P=0.36)$.

\section{Clinical investigation}

Clinical examination was carried out by a physician $(\mathrm{VH}$, HL, JR) at the Turku PET Centre, University of Turku, Turku, Finland, between June 8, 2012, and April 29, 2014. It included measurements of weight, height, waist and hip circumference, and total body fat \% (Omron HBF-400-E) In addition, study subjects kept an exercise diary on leisure-time physical activity during the intervention and the follow-up. In brief, frequency of leisure-time physical activity per week was $5.3 \pm 2.9$ during the intervention and $5.2 \pm 2.4$ during the follow-up $(P=0.50)$. Amount of leisure-time physical activity was $55.6 \pm 32.1 \mathrm{~min}$ per session during the intervention and $60.9 \pm 32.2 \mathrm{~min}$ per session during the follow-up $(P=0.19)$.

\section{CT studies}

CTXA of the hip from left proximal femur in $\mathrm{g} / \mathrm{cm}^{2}$ was measured using quantitative CT (Discovery 690, General Electric (GE) Medical systems) with a resolution of $3.75 \mathrm{~mm}$. A region located between the superior aspect of the femoral head and inferior aspect of the lesser trochanter was scanned with the patient lying in a supine position on the CT table. The CT values were converted to BMD scale using a solid calibration phantom (Mindways) placed underneath the patients during the scan. All scans were performed at SFOV $500 \mathrm{~mm}$, pitch $1.375,120 \mathrm{kV}$ and $79 \mathrm{mAs}$. Images were transferred to a QCT workstation and analyzed using the CTXA hip function version 4.2.3 of Mindways QCT Pro software (Mindways Software Inc., Austin, TX, USA). In the analysis of hip, isolation and rotation of the hip were performed. QCT Pro uses a default threshold value of $100 \mathrm{mg} / \mathrm{cm}^{3}$ to separate bone from soft tissue. Femoral neck axis was identified automatically with the Mindways Optimize FN Axis algorithm. A rectangular femoral neck 
Table 1 Baseline characteristics of subjects included in and excluded from follow-up. Results are presented as mean \pm s.D.

\begin{tabular}{|c|c|c|c|}
\hline & Included $(n=25)$ & Excluded $(n=23)$ & $\boldsymbol{P}$ \\
\hline Age (years) & $71.8 \pm 2.9$ & $72.6 \pm 3.1$ & 0.35 \\
\hline Handgrip strength $(\mathrm{kg})^{\dagger}$ & $19.9 \pm 5.7$ & $20.6 \pm 5.5$ & 0.64 \\
\hline Body mass index $\left(\mathrm{kg} / \mathrm{m}^{2}\right)$ & $27.9 \pm 4.6$ & $26.3 \pm 4.5$ & 0.23 \\
\hline Waist-to-hip ratio & $0.91 \pm 0.05$ & $0.90 \pm 0.06$ & 0.63 \\
\hline Total body fat $(\%)$ & $40.6 \pm 5.1$ & $37.9 \pm 6.1$ & 0.11 \\
\hline Suppl. vitamin D intake ( $\mu \mathrm{g} / \mathrm{day})$ & $3.6 \pm 9.5$ & $3.0 \pm 7.0$ & 0.82 \\
\hline Suppl. calcium intake (mg/day) & $120.0 \pm 298.6$ & $152.2 \pm 351.5$ & 0.73 \\
\hline Vitamin D and calcium suppl. in use (\%) & 16.0 & 17.4 & 0.99 \\
\hline Earlier diagnosed osteoporosis (\%) & 16.0 & 8.7 & 0.67 \\
\hline Current HRT (\%) & 12.0 & 4.3 & 0.61 \\
\hline 10 -year RR for MO fracture (\%) & $5.46 \pm 1.95$ & $5.75 \pm 2.54$ & 0.83 \\
\hline 10-year RR for hip fracture (\%) & $1.04 \pm 0.92$ & $1.28 \pm 1.41$ & 0.83 \\
\hline Total hip BMD $\left(\mathrm{g} / \mathrm{cm}^{2}\right)$ & $0.695 \pm 0.110$ & $0.709 \pm 0.126$ & 0.68 \\
\hline Femoral neck BMD $\left(\mathrm{g} / \mathrm{cm}^{2}\right)$ & $0.723 \pm 0.153$ & $0.725 \pm 0.178$ & 0.96 \\
\hline Trochanteric BMD $\left(\mathrm{g} / \mathrm{cm}^{2}\right)$ & $0.609 \pm 0.113$ & $0.604 \pm 0.114$ & 0.87 \\
\hline Intertrochanteric BMD $\left(\mathrm{g} / \mathrm{cm}^{2}\right)$ & $0.822 \pm 0.160$ & $0.821 \pm 0.203$ & 0.98 \\
\hline Quadriceps m. mass (g) & $144.5 \pm 22.1$ & $144.6 \pm 20.7$ & 0.99 \\
\hline Adductor magnus $\mathrm{m}$. mass $(\mathrm{g})$ & $96.4 \pm 19.0$ & $92.7 \pm 13.6$ & 0.44 \\
\hline Hamstring $\mathrm{m}$. mass $(\mathrm{g})$ & $49.7 \pm 14.4$ & $50.3 \pm 7.3$ & 0.85 \\
\hline Adductor longus $\mathrm{m}$. mass ( $\mathrm{g}$ ) & $26.5 \pm 8.9$ & $24.3 \pm 4.4$ & 0.28 \\
\hline CTX-I (ng/mL) & $0.44 \pm 0.23$ & $0.43 \pm 0.25$ & 0.92 \\
\hline PINP (ng/mL) & $49.0 \pm 24.0$ & $45.2 \pm 20.6$ & 0.56 \\
\hline Total osteocalcin (ng/mL) & $8.5 \pm 4.0$ & $8.8 \pm 3.9$ & 0.81 \\
\hline Serum sclerostin (pmol/L) & $55.9 \pm 15.5$ & $71.7 \pm 19.0$ & $0.004 *$ \\
\hline IGF-I (ng/mL) & $90.0 \pm 27.7$ & $77.7 \pm 21.6$ & 0.74 \\
\hline 25(OH)-D (nmol/L) & $71.8 \pm 28.1$ & $83.1 \pm 30.6$ & 0.19 \\
\hline
\end{tabular}

${ }^{*} P<0.05 ;{ }^{\dagger}$ Assessed in 2001-2004. HRT, hormonal replacement therapy; MO, major osteoporotic; BMD, bone mineral density.

ROI with thickness of $15 \mathrm{~mm}$ was used, and it was placed in the femoral collum resting on top of the trochanters excluding femoral head. The distal extent line was set to the base of the lesser trochanter (Fig. 2). The trochanteric and intertrochanteric BMD were assessed automatically. BMD measurements were obtained for 36, 32 and 19 intervention subjects and for 11 and 6 control subjects, respectively. One baseline study and two post-intervention studies were cancelled due to technical difficulties. One post-intervention study was cancelled owing to withdrawal of consent and two intervention subjects did not participate in the intervention. Baseline and post-intervention BMD measurements were analyzed after the post-intervention visit. BMD analyses were performed by a single investigator $(\mathrm{VH})$.

The 10-year RR for major osteoporotic (MO) and hip fracture was calculated with the WHO Fracture Risk Assessment Tool (http://www.shef.ac.uk/FRAX/). After the post-interventional analyses, calcium (dosage $1000 \mathrm{mg}$ / day) and vitamin D supplement enhancements (dosage $20 \mu \mathrm{g}$ /day) were recommended to $76 \%$ of subjects included in the follow-up based on the QCT results. The self-reported use of calcium dosage range of all follow-up subjects was $0-1000 \mathrm{mg} /$ day and vitamin D dosage range was $0-60 \mu \mathrm{g} /$ day assessed with a questionnaire at the follow-up visit.
Methodology for obtaining muscle masses is described in the supplementary material of our previous article by Bucci et al. (28). As short, the thigh CT images were semiautomatically co-registered to the whole-body MRI images and then measures were taken to select a $1.5 \mathrm{~cm}$ thick section $(1.6 \mathrm{~cm}$ mask in the imaging software) that was at $5 \mathrm{~cm}$ distance (perpendicularly) from the pubic region (with right and left inguinal lines showed in coronal view). It followed the manual segmentation of the different muscle groups by drawing ROIs in the 5-6 selected slices in the Carimas software. The selected compartments were quadriceps, adductor magnus, hamstring and adductor longus muscles. After drawing the ROIs surrounding the muscle groups, the muscle tissue volume on the CT image was segmented via HU thresholding. A threshold of 0 to $100 \mathrm{HU}$ was used as described previously (30). The different muscle group volumes were converted into masses using the skeletal muscle density $\left(1.04 \mathrm{~g} / \mathrm{cm}^{3}\right)$.

\section{Bone markers}

Fasting morning serum samples were collected at baseline and after the 16-week intervention. The third sample was 
Table 2 Baseline characteristics of the study groups. Results are presented as mean \pm S.D.

\begin{tabular}{|c|}
\hline \multirow{2}{*}{$\begin{array}{l}\text { Age (years) } \\
\text { Handgrip strength }(\mathrm{kg})^{\dagger}\end{array}$} \\
\hline \\
\hline Body mass index $\left(\mathrm{kg} / \mathrm{m}^{2}\right)$ \\
\hline Waist to hip ratio \\
\hline Total body fat (\%) \\
\hline Suppl. vitamin D intake ( $\mu \mathrm{g} /$ day) \\
\hline Suppl. calcium intake (mg/day) \\
\hline Vitamin D and calcium suppl. in use (\%) \\
\hline Earlier diagnosed osteoporosis (\%) \\
\hline Current HRT (\%) \\
\hline 10 -year RR for MO fracture (\%) \\
\hline 10 -year RR for hip fracture (\%) \\
\hline Total hip BMD $\left(\mathrm{g} / \mathrm{cm}^{2}\right)$ \\
\hline Femoral neck BMD $\left(\mathrm{g} / \mathrm{cm}^{2}\right)$ \\
\hline Trochanteric BMD $\left(\mathrm{g} / \mathrm{cm}^{2}\right)$ \\
\hline Intertrochanteric BMD $\left(\mathrm{g} / \mathrm{cm}^{2}\right)$ \\
\hline Quadriceps m. mass (g) \\
\hline Adductor magnus $\mathrm{m}$. mass (g) \\
\hline Hamstring $\mathrm{m}$. mass $(\mathrm{g})$ \\
\hline Adductor longus $\mathrm{m}$. mass ( $\mathrm{g}$ ) \\
\hline CTX-I (ng/mL) \\
\hline PINP (ng/mL) \\
\hline Total osteocalcin (ng/mL) \\
\hline Serum sclerostin (pmol/L) \\
\hline IGF-I (ng/mL) \\
\hline 25(OH)-D (nmol/L) \\
\hline
\end{tabular}

\begin{tabular}{c}
\hline Control $(n=11)$ \\
\hline $71.8 \pm 2.9$ \\
$29.6 \pm 2.5$ \\
$26.8 \pm 4.4$ \\
$0.89 \pm 0.04$ \\
$38.8 \pm 5.3$ \\
$3.2 \pm 8.8$ \\
$181.8 \pm 337.1$ \\
27.3 \\
9.1 \\
9.1 \\
$5.46 \pm 1.95$ \\
$1.04 \pm 0.92$ \\
$0.740 \pm 0.118$ \\
$0.724 \pm 0.130$ \\
$0.657 \pm 0.106$ \\
$0.890 \pm 0.193$ \\
$157.8 \pm 22.0$ \\
$100.0 \pm 17.1$ \\
$52.4 \pm 11.9$ \\
$26.1 \pm 6.4$ \\
$0.37 \pm 0.15$ \\
$40.8 \pm 10.0$ \\
$7.4 \pm 2.8$ \\
$74.9 \pm 19.6$ \\
$86.4 \pm 25.1$ \\
$84.8 \pm 40.9$ \\
\end{tabular}

\begin{tabular}{c}
\hline Intervention $(n=37)$ \\
\hline $71.9 \pm 3.1$ \\
$17.7 \pm 2.6$ \\
$27.2 \pm 4.7$ \\
$0.91 \pm 0.05$ \\
$39.5 \pm 5.9$ \\
$3.6 \pm 6.7$ \\
$121.6 \pm 320.8$ \\
13.5 \\
13.5 \\
8.1 \\
$5.75 \pm 2.54$ \\
$1.28 \pm 1.41$ \\
$0.690 \pm 0.115$ \\
$0.724 \pm 0.174$ \\
$0.591 \pm 0.111$ \\
$0.801 \pm 0.173$ \\
$140.5 \pm 19.5$ \\
$92.9 \pm 16.2$ \\
$49.2 \pm 11.2$ \\
$25.2 \pm 7.4$ \\
$0.46 \pm 0.25$ \\
$49.0 \pm 24.6$ \\
$9.0 \pm 4.1$ \\
$60.8 \pm 17.9$ \\
$83.4 \pm 25.9$ \\
$74.9 \pm 25.6$ \\
\end{tabular}

\begin{tabular}{c}
\hline \multicolumn{1}{c}{} \\
\hline 0.79 \\
$<0.001 * *$ \\
0.82 \\
0.44 \\
0.73 \\
0.40 \\
0.35 \\
0.36 \\
0.70 \\
0.92 \\
0.83 \\
0.83 \\
0.26 \\
0.87 \\
0.09 \\
0.15 \\
$0.016 *$ \\
0.22 \\
0.42 \\
0.70 \\
0.27 \\
0.27 \\
0.23 \\
$0.037 *$ \\
0.74 \\
0.34 \\
\end{tabular}

${ }^{*} P<0.05 ;{ }^{*} P<0.001 ;{ }^{\dagger}$ Assessed in 2001-2004. HRT, hormonal replacement therapy; MO, major osteoporotic; BMD, bone mineral density.

collected at follow-up visit. Serum samples were stored as aliquots at $-80^{\circ} \mathrm{C}$. Serum samples were obtained for 37,34 and 19 intervention subjects. In the control group, serum samples were collected at baseline and at the follow-up visit. Serum samples were available for 11 and 6 controls, respectively. All samples were measured as duplicates and simultaneously at the end of the study.

Bone resorption was assessed by C-terminal crosslinked telopeptides of type I collagen (CTX, IDS-iSYS CTX-I ELISA, CrossLaps ${ }^{\circledR}$ ) and bone formation by intact $\mathrm{N}$-terminal propeptide of type I collagen (PINP, IDSiSYS IntacPINP assay). We also measured serum levels of insulin-like growth factor-I (IGF-I) with IDS-iSYS Insulinlike Growth Factor-I assay. All iSYS assays were purchased from IDS Ltd, UK, and measurements were performed at ValiRx Finland Ltd (Oulu, Finland). Plasma levels of 25-hydroxyvitamin D (25(OH)-D) were measured with the Elecsys ${ }^{\circledR}$ Vitamin D Total immunoassay (Roche Diagnostics $\mathrm{GmbH}$ ). Serum total osteocalcin (TotalOC) was determined with two-site immunoassay based on monoclonal antibodies 2H9 and 6F9 using previously described protocol (31). Serum sclerostin was measured with Sclerostin ELISA from Biomedica (Vienna, Austria) according to manufacturer's instructions. Two subjects and one control had serum sclerostin at baseline below the detection limit $(<25 \mathrm{pmol} / \mathrm{L})$ and sclerostin data was thus obtained only for 35 subjects and 10 controls at baseline.

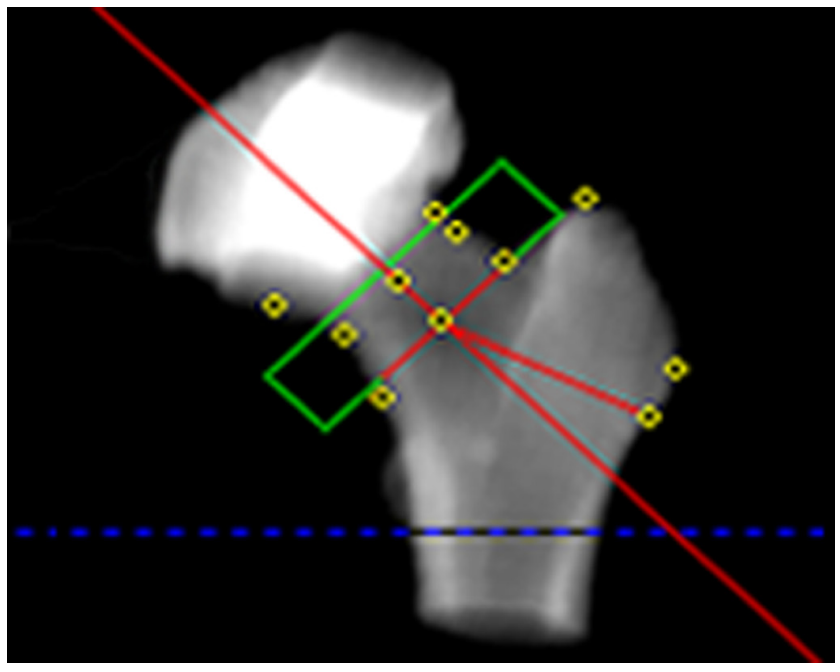

\section{Figure 2}

Positioning of femoral neck region of interest (green rectangle) and distant extent line (blue dashed line). 


\section{Statistical methods}

Statistical analyses were performed using SPSS software for Mac OS (version 22.00). Data is presented as mean \pm S.D.. The Shapiro-Wilk test was used to assess the normality of variables. Differences among the groups were tested with independent samples $t$-test or Mann-Whitney $U$ test if data was not normally distributed. Differences among categorical data were tested with chi-square test. Differences between pre-intervention, post-intervention and follow-up visit parameters were assessed with repeated measures ANOVA with unstructured covariance structure. Bonferroni adjusted $P$ values were used in pairwise comparisons between the time points. Associations of $\mathrm{BMD}$, the main outcome variable with other variables were tested with Spearman's correlation. $P$ value less than 0.05 was considered statistically significant.

\section{Results}

\section{Baseline characteristics}

Handgrip strength assessed during 2001 to 2004 was lower in the intervention group $(P<0.001)$. Age, body mass index, waist-to-hip ratio, total body fat, supplemental calcium and vitamin D intake did not differ between the groups. About $13.5 \%$ of intervention subjects and $27.3 \%$ of control subjects used calcium and/or vitamin D supplements $(P=\mathrm{NS})$. No differences were found in the prevalence of earlier diagnosis of osteoporosis, percent of subjects currently using HRT or 10-year RR for MO or hip fracture between the groups. Total hip, femoral neck, trochanteric or intertrochanteric BMD did not differ between the groups. Quadriceps muscle mass was lower in the intervention group compared with control group $(P=0.016)$. No differences in adductor longus, adductor magnus or hamstring muscle masses were found. The levels of bone turnover markers were similar in the intervention and control groups with the exception of sclerostin, which was slightly higher in control group $(P=0.037)$ (Table 2).

\section{Changes in muscular strength and mass}

Resistance training increased mean quadriceps muscle mass by $9.2 \%(P=0.001)$ and adductor magnus muscle mass by $4.4 \%(P=0.012)$ as reported previously by Bucci et al. (28). No increase in hamstring or adductor longus muscle masses was observed. No correlations were observed between the changes in thigh muscle masses and the change in hip BMD.
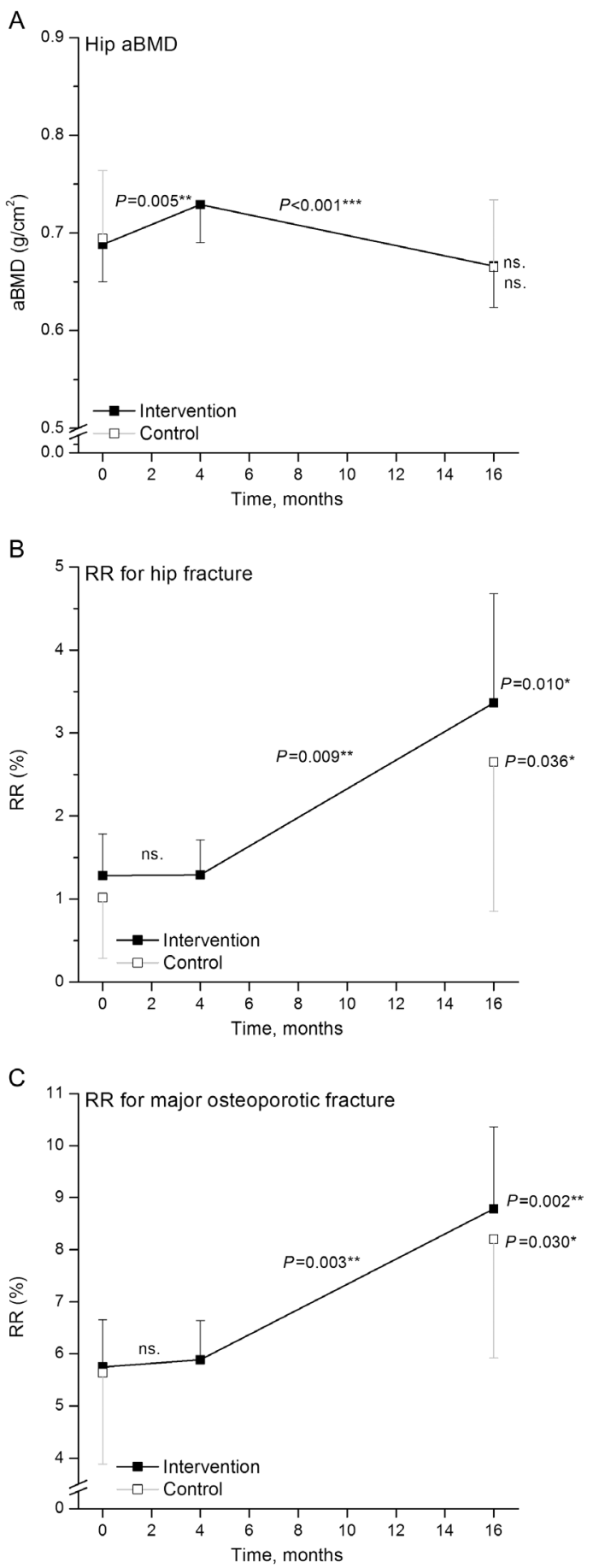

\section{Figure 3}

Longitudinal changes in total hip BMD, 10-year relative risk for hip and major osteoporotic fracture. (A) Total hip BMD,

(B) 10-year relative risk for hip fracture and (C) 10-year relative risk for major osteoporotic fracture at baseline, after the 16-week resistance training and 1 year after the cessation of the resistance training. Results are shown as estimated means with $95 \% \mathrm{Cl} .{ }^{*} P<0.05, * * P<0.01, * * * P<0.001$. 
Table 3 Bone marker concentrations at pre-intervention, post-intervention and follow-up visits.

\begin{tabular}{l}
\hline \\
\hline CTX (ng/mL) \\
PINP (ng/mL) \\
Total osteocalcin (ng/mL) \\
Serum sclerostin (pmol/L) \\
IGF-1 (ng/mL) \\
25(OH)-D (nmol/L) \\
\hline
\end{tabular}

\begin{tabular}{|c|c|}
\hline & Intervention \\
\hline Pre $(n=37)$ & Post $(n=34)$ \\
\hline $0.46(0.38 ; 0.54)$ & $0.46(0.36 ; 0.55)$ \\
\hline $49.0(40.8 ; 57.2)$ & $49.2(41.7 ; 56.7)$ \\
\hline $9.0(7.6 ; 10.4)$ & $10.3(8.6 ; 12.1)^{\dagger}$ \\
\hline $60.9(54.1 ; 67.6)$ & $84.3(77.4 ; 91.1) *$ \\
\hline $83.4(74.8 ; 92.1)$ & 85.7 (77.9; 93.6) \\
\hline $74.9(66.4 ; 83.5)$ & 77.1 (68.2; 86.4) \\
\hline
\end{tabular}

Results are denoted as estimated means with $95 \% \mathrm{Cl}$.

${ }^{\S} P<0.001$ vs pre and post, ${ }^{\S \S} P=0.006$ vs pre.

${ }^{\dagger} P=0.04$ vs pre, ${ }^{\dagger+} P=0.02$ vs pre.

$* P<0.001$ vs pre, $* * P=0.45$ vs post.

${ }^{\ddagger} P<0.001$ vs pre and post, ${ }^{\ddagger \ddagger} P=0.002$ vs pre.

Muscle strength measured by mean RM on 8 (RM8 in $\mathrm{kg}$ ) increased by $60 \%$ on the leg press, $96 \%$ on seated row, $118 \%$ on the chest press, $51 \%$ on abdominal crunches, $70 \%$ on seated leg curls, $69 \%$ on back extensions and $43 \%$ on hip abduction after the resistance training intervention $(P<0.001$ for all). No significant correlations were observed between the change in measured muscle group strength and BMD. BMI, waist-to-hip ratio and total body fat percentage remained unchanged after the intervention.

\section{Changes in BMD, 10-year RR for osteoporotic fracture and bone markers}

Resistance training seemed to increase the total hip BMD by a mean of $6 \%(P=0.005)$ (Fig. 3). The change in the total hip BMD was mainly due to increased bone mass in the intertrochanteric area $(+10.7 \%, P=0.001)$, while the BMD of trochanteric $(+2.0 \%, P=\mathrm{NS})$ and femoral neck $(-4.1 \%$, $P=$ NS) areas remained unchanged. Serum levels of sclerostin $(P<0.001)$ and total osteocalcin $(P=0.04)$ increased after the intervention (Fig. 3) while the levels of CTX $(P=\mathrm{NS})$, PINP $(P=\mathrm{NS})$, IGF-1 $(P=\mathrm{NS})$ and 25(OH)-D $(P=\mathrm{NS})$ remained unchanged (Table 3). The 10-year RR for hip fracture remained unchanged ( $P=\mathrm{NS}$ ) (Fig. 3). No correlations between the changes in bone markers and changes in BMD were observed (data not shown).

\section{Changes in BMD and bone markers during the follow-up}

Mean total hip BMD decreased by $8.6 \%(P<0.001)$ in the intervention group at follow-up when compared with post-intervention values. In controls, total hip BMD $(P=\mathrm{NS})$ remained unchanged (Fig. 3). Serum sclerostin decreased in the intervention group $(P=0.045)$ but serum osteocalcin remained unchanged $(P=\mathrm{NS})$. 25(OH)-D increased after the intervention $(P<0.001)$ and CTX decreased $(P<0.001)$ but PINP $(P=\mathrm{NS})$ and IGF-1 $(P=\mathrm{NS})$ remained unchanged (Table 3). The 10-year RR for hip fracture increased by $2.1 \%(P=0.01)$ and for MO fracture by $3.0 \%(P=0.002)$ in the intervention group at follow-up when compared with pre-intervention values (Fig. 3). In controls, 25(OH)-D $(P=0.002)$ and total osteocalcin $(P=0.02)$ increased but CTX decreased $(P=0.006)$. Serum sclerostin $(P=\mathrm{NS}), \quad$ PINP $(P=\mathrm{NS})$ and IGF-1 $(P=\mathrm{NS})$ remained unchanged (Table 3). The 10-year RR for hip fracture increased by $1.6 \%(P=0.036)$ and for $\mathrm{MO}$ increased by $2.6 \%(P=0.03)$ at follow-up compared with pre-intervention values (Fig. 3).

Mean calcium $(121.6 \pm 320.7$ vs $842.1 \pm 374.6 \mathrm{mg} /$ day) and vitamin $\mathrm{D}$ supplement intake $(3.24 \pm 8.8$ vs $26.8 \pm 14.0 \mu \mathrm{g} /$ day) increased in the intervention group $(P<0.001$ in both $)$. In controls, vitamin D $(8.2 \pm 7.3$ vs $27.0 \pm 13.0 \mu \mathrm{g} / \mathrm{day}, P=0.03)$ but not calcium $(222.2 \pm 363.2$ vs $600 \pm 547.7 \mathrm{mg} /$ day, $P=\mathrm{NS}$ ) supplement intake increased. There was no correlation between the decrease in total hip BMD and vitamin D and calcium supplement intake or increase in $25(\mathrm{OH})$-D during the follow-up. In addition, no correlation was observed between increase in $25(\mathrm{OH})$-D and decrease in CTX. BMI, waist-to-hip ratio and total body fat remained unchanged in intervention and control groups during the entire follow-up period (data not shown).

\section{Discussion}

We studied the effects of a 16-week resistance training regimen on BMD, serum bone markers and 10-year RR for osteoporotic fracture in elderly women with decreased muscle strength. It appears that resistance training significantly increased total hip BMD as well as concentrations of serum sclerostin and osteocalcin. The 
10-year RR for osteoporotic fracture remained unchanged. Moreover, we found that total hip BMD decreased back to baseline levels and the 10-year RR for osteoporotic fracture increased significantly after discontinuation of the resistance training intervention. Bone markers, except for serum sclerostin, CTX and 25(OH)-D, remained unchanged after 1 year from the end of the intervention. Our findings suggest a beneficial role of continuous supervised resistance training for the prevention of osteoporotic fractures.

The 16-week resistance training intervention increased mean total hip BMD, but 10-year RR for osteoporotic fracture remained unchanged. This is explained by the fact that the FRAX calculation is based on femoral neck BMD (21) that remained unchanged in our study. The reason why femoral neck BMD did not increase may be explained by the lack of a site-specific muscle-bone interaction, which is discussed later on. Nonetheless, the finding that total hip BMD increased in response to resistance exercise seems to be in line with the only longitudinal QCT study reported previously. Lang et al. found that total hip BMD increased by 3.4\% after 16-week resistance training intervention in 25 to 55 -year-old male and female subjects (32). We found a $6 \%$ increase in total hip BMD, which is higher compared with the finding by Lang et al. However, this may result from a different age group, gender or muscle strength of study subjects or differences in exercise intervention protocols.

An increase in BMD due to resistance training has been reported to be lower in longitudinal studies in which BMD was assessed with DXA compared with QCT. Bemben et al. found that a 40-week resistance training regimen, regardless of intensity and frequency, was effective to improve hip BMD up to $1.5 \%$ in elderly male and female subjects (10). Marques et al. found an 8-month resistance training regimen increased proximal femur BMD up to $2.9 \%$ in elderly women (11). The magnitude in BMD changes found may be explained by differences in the intensity of resistance training interventions or in demographic profiles of subjects as well as in methodology, that is, QCT and DXA combined with inter- and intra-observer precision errors. In our study, the same experienced investigator carried out all the QCT analyses, which means that inter-observer reproducibility was not evaluated. Intra-observer precision errors for our method for femoral neck BMD have been reported to be approximately $1.5 \%$ (33). Moreover, Lang et al. have shown that the precision of duplicate QCT scans of cadaveric femurs was excellent (34). It has also been found that the precision of CTXA duplicate hip scans are slightly better than DXA (25), which may be explained with the fact that QCT intra-observer precision error is caused mainly by image isolation and rotation of the hip during the analyses. Overall, we conclude that differences in the BMD increases among the studies are likely explained by methodological differences, but it is not excluded that these increases may also be more prominent in subjects with decreased muscle strength which would indicate a connection between muscles and bone.

We found no correlations between muscle strength and BMD. However, muscle-bone interaction may still underlie the BMD outcome, which may be partly explained by spatially heterogeneous response of bone to resistance training targeted to different muscles (32). This is supported by Marques et al. who suggested that the positive effect of exercise observed in the total hip BMD may be related with the inclusion of movements, such as hip abduction stimulating the gluteus muscles, which insert in the greater trochanter (11). In our study, the increase in hip BMD was mainly located in the intertrochanteric area, which is the origin site of vastus muscles of quadriceps femoris. Moreover, we found that the mass of this muscle group increased after exercise, but the correlation between an increase in thigh muscle masses and total hip BMD was not observed. Ma et al. (35) studied the muscle-bone unit in subjects with varying BMD and found that in the subjects with osteopenia or osteoporosis, relatively larger muscle masses act on the weakened bones independent of age, which in other words means that as BMD decreases abnormally, more bone than muscle is lost. In short, we found that BMD increased simultaneously with muscle mass without observed correlation. This may indicate that increased muscle mass results in increased mechanical strain on bone that stimulates site-specific osteogenesis through osteoblast and osteocyte activation.

Serum sclerostin, an osteocyte-specific protein that inhibits bone formation via Wnt-signaling pathway (17), and muscle strength were lower in the intervention group compared with controls at baseline. The Wnt-signaling pathway is known to inhibit muscle differentiation (36), and this may be one explanation for the difference found in the serum sclerostin concentration. A similar finding has been made in young female gymnasts who had higher serum sclerostin levels compared with controls (37). In addition, we found that hip BMD distribution between controls and the intervention group was different. This may be associated with a difference in muscle strength or serum sclerostin levels among the groups. Shen et al. (38) found that the osteocyte-specific ablation of $C x 43$ gene 
not only reduced BMD, but also had a negative effect on muscle mass in mice, which suggests a link between osteocytes and surrounding muscles.

We found that serum sclerostin levels increased significantly after resistance training. Given that sclerostin locally inhibits bone formation, this is not in agreement with the osteogenic effect observed in our study. Circulating sclerostin levels have been shown to correlate closely with bone marrow levels (17). However, it is not yet elucidated how well the changes in circulating sclerostin reflect the changes in bone microenvironment. The sources of biological variability are still unclear, and circulating sclerostin levels do not always correlate as expected with observed BMD (17). Similar findings have earlier been made in elderly women with anti-osteoporotic medication. Polyzos et al. found that sclerostin levels increased with BMD after 6-month risedronate treatment (18), although decrease was expected. Moreover, limited data is available concerning serum sclerostin and physical activity in humans. Falk et al. observed an acute increase in serum sclerostin levels in young men following one session of high-impact exercise (39). Lombardi et al. compared sclerostin serum concentrations among athletes belonging to different sports characterized by different weight-bearing exercises. They found that serum sclerostin was higher in weight-bearing than in non-weight-bearing subjects (40). Increased circulating levels of sclerostin may reflect the increase in muscle mass or activation of sclerostin-synthesizing osteocytes (17) that may be caused by resistance training-induced mechanostimulation or bone microdamage (41). It is not ruled out that exercise-induced effects on serum sclerostin levels are age and sex dependent. In contrast to earlier studies (18-20), we found no correlation between serum sclerostin and BMD, which suggests that serum sclerostin levels do not always correlate with BMD (17). Circulating levels of osteocalcin also increased after resistance training, which may indicate the activity of osteocalcinexpressing osteoblasts and osteocytes and increased bone formation/bone remodeling in response to exerciseinduced mechanostimulation or bone microdamage.

To our knowledge, this is the first study to assess BMD at follow-up 1 year after discontinuation of the resistance training intervention. We found that total hip BMD returned approximately to baseline levels. This finding highlights the importance of continuous supervised resistance training in maintaining the achieved BMD. Engelke et al. found in their follow-up study that total hip BMD decreased significantly by $1 \%$ at the 1 -year time point in a control group without previous exercise interventions (4). In our study, the decrease in BMD was more rapid. The typical average BMD loss is $1.0-3.7 \%$ per year in non-exercising postmenopausal women (42). It should be noted that the expected decrease in BMD was not observed in non-exercising controls in our study, which likely results from low statistical power within the control group. Simultaneous vitamin D and calcium supplemental use has been found to lead to a small increase in BMD in postmenopausal women (43). This was not the case in our study because total hip BMD decreased despite the increases in vitamin $\mathrm{D}$ and calcium supplemental intake and 25(OH)-D concentration. We found no correlation between decrease in total hip BMD and vitamin D or calcium supplement intake or between decrease in total hip BMD and increase in 25(OH)-D during the follow-up suggesting that calcium and vitamin $\mathrm{D}$ supplementation or increase in $25(\mathrm{OH})$-D concentration did not probably affect the observed decrease in BMD at the follow-up.

CTX decreased similarly in both intervention and controls groups at the follow-up. One possible explanation to this may be simultaneous increase in $25(\mathrm{OH})-\mathrm{D}$, which may be caused by the increase in vitamin $\mathrm{D}$ supplement intake in both groups. Inverse correlations between 25(OH)-D and CTX concentrations have earlier been reported in obese subjects (44) and in postmenopausal women (45). However, in our study no correlation between change in CTX and change in 25(OH)-D concentrations was found which is probably due to too low sample size. Osteocalcin levels remained elevated at the follow-up visit, which suggests that a decrease in osteocalcin back to baseline levels would require more time compared with the decrease in BMD. Interestingly, we found that serum sclerostin levels decreased after cessation of resistance training intervention. Taken together, these two findings could also suggest that osteocalcin levels remain elevated due to high bone remodeling also during the phase of bone loss after cessation of training, while the levels of sclerostin reflect the exercise-induced activation of osteocytes. To our knowledge, studies investigating the long-term changes in serum sclerostin levels, after cessation of resistance training intervention, have not been conducted.

Strengths of this study included a well-characterized study population, the assessment of serum bone markers simultaneously with high-quality CTXA hip BMD measurements. The resistance training intervention was efficient, as muscle strength and muscle masses increased. This reflects the adherence and motivation of our study population. In addition, subjects kept exercise diaries during the intervention and the follow-up period. We found no differences in frequency or amount of leisure- 
time physical activity between the intervention and follow-up period. This means that leisure-time physical activity did not probably have an effect on our results. A major limitation of our study was a relatively small sample size. In addition, controls were not re-examined after 16 weeks simultaneously with the intervention group, which may mean that BMD gain of $6 \%$ by technical causes cannot be completely ruled out. In addition, the follow-up consisted only of a subset of subjects, thus decreasing the statistical power. We did not have the possibility to analyze cortical and trabecular BMD simultaneously due to lack of advanced research tools. An additional limitation was lack of information about the age of menarche, years after menopause, parity and previous HRT treatment of the study subjects, possibly influencing the external validity of our results.

Our control group had normal muscle strength, which may seem to be a flaw in the study design. Original purpose of the control group was to demonstrate that the intervention group truly had decreased muscle strength at baseline. However, we did not test the effects of exercise or follow-up between the intervention and control group but only within the groups. One limitation in our study was that dietary intakes of vitamin D and calcium were not assessed. However, supplemental vitamin D and calcium intake data was available and adherence to this treatment seemed to be excellent because 25(OH)-D increased at the follow-up in both groups. Supplemental intakes were reported to be low at baseline probably because only minority of subjects used them. Nonetheless, supplemental vitamin D intake increased in both groups but calcium intake increased only in intervention group. It may be that significant increase of calcium intake in control group was not observed because of too small sample size. Another limitation in our study was poor follow-up, which consisted only approximately 50\% of the subjects. However, all the subjects, excluding those who were prescribed an anti-osteoporotic treatment (other than calcium and vitamin D) were invited to follow-up visit. One explanation for poor follow-up may be low willingness to join the follow-up study after very intense interventional study. Comparison of the baseline characteristics between included and excluded subjects did not show any differences except for serum sclerostin. This difference persisted even after adjusting for muscle strength and the reason for this remains unknown.

In conclusion, we found that 16-week resistance training intervention seemed to increase BMD and maintained the 10-year RR for fracture in elderly women with decreased muscle strength. These positive outcomes were not sustained 1 year after the cessation of resistance training intervention. These findings suggest a beneficial role of continuous supervised resistance training in the prevention of osteoporotic fractures in elderly women with decreased muscle strength.

\section{Declaration of interest}

The authors declare that there is no conflict of interest that could be perceived as prejudicing the impartiality of the research reported.

\section{Funding}

This work was supported by funding from the DORIAN EU FP7 (project number 278603, DORIAN: Developmental ORIgins of healthy and unhealthy AgeiNg: The role of maternal obesity), Turku University Foundation, Medical Imaging Centre of Southwest Finland and Turku University Hospital, Radiological Society of Finland and Foundation of Uulo Arhio, Finnish Cultural Foundation, Oskar Öflund Foundation, Research Foundation of Clinical Chemistry, Finnish Medical Foundation, The Academy of Finland, Sigrid Juselius Foundation and the Emil Aaltonen Foundation.

\section{Acknowledgements}

We thank the staff of the Turku PET Centre and Turku University Central Hospital for performing PET and CT imaging and laboratory analyses. We also thank the study nurses M Koutu and P Nyholm for their assistance, Folkhälsan Research Center physiotherapists L Penttinen and M Lipasti who carried out the resistance training intervention and T Vahlberg for his statistical expertise. We thank Aura Professional English Consulting, Ltd. for the language editing of this manuscript.

\section{References}

1 Kanis JA, McCloskey EV, Johansson H, Cooper C, Rizzoli R, Reginster JY \& Scientific Advisory Board of the European Society for Clinical and Economic Aspects of Osteoporosis and Osteoarthritis (ESCEO) and the Committee of Scientific Advisors of the International Osteoporosis Foundation (IOF). European guidance for the diagnosis and management of osteoporosis in postmenopausal women. Osteoporosis International 201324 23-57. (doi:10.1007/ s00198-012-2074-y)

2 McClung MR, Geusens P, Miller PD, Zippel H, Bensen WG, Roux C, Adami S, Fogelman I, Diamond T, Eastell R et al. Effect of risedronate on the risk of hip fracture in elderly women. Hip Intervention Program Study Group. New England Journal of Medicine $20013 \mathbf{3 4 4}$ 333-340. (doi:10.1056/NEJM200102013440503)

3 Bocalini DS, Serra AJ, dos Santos L, Murad N \& Levy RF. Strength training preserves the bone mineral density of postmenopausal women without hormone replacement therapy. Journal of Aging and Health 200921 519-527. (doi:10.1177/0898264309332839)

4 Engelke K, Kemmler W, Lauber D, Beeskow C, Pintag R \& Kalender WA. Exercise maintains bone density at spine and hip EFOPS: a 3-year longitudinal study in early postmenopausal women. Osteoporosis International 200617 133-142. (doi:10.1007/s00198-0051938-9)

5 Nelson ME, Fiatarone MA, Morganti CM, Trice I, Greenberg RA $\&$ Evans WJ. Effects of high-intensity strength training on multiple risk factors for osteoporotic fractures. A randomized controlled trial. JAMA 1994272 1909-1914. (doi:10.1001/ jama.1994.03520240037038) 
6 Taaffe DR, Duret C, Wheeler S \& Marcus R. Once-weekly resistance exercise improves muscle strength and neuromuscular performance in older adults. Journal of the American Geriatrics Society 199947 1208-1214. (doi:10.1111/j.1532-5415.1999.tb05201.x)

7 Liu CJ \& Latham NK. Progressive resistance strength training for improving physical function in older adults. Cochrane Database of Systematic Reviews 20098 CD002759. doi CD002759. (doi:10.1002/14651858.cd002759.pub2)

8 Taaffe DR, Pruitt L, Pyka G, Guido D \& Marcus R. Comparative effects of high- and low-intensity resistance training on thigh muscle strength, fiber area, and tissue composition in elderly women. Clinical Physiology 199616 381-392. (doi:10.1111/j.1475-097X.1996. tb00727.x)

9 Rhodes EC, Martin AD, Taunton JE, Donnelly M, Warren J \& Elliot J. Effects of one year of resistance training on the relation between muscular strength and bone density in elderly women. British Journal of Sports Medicine 200034 18-22. (doi:10.1136/ bjsm.34.1.18)

10 Bemben DA \& Bemben MG. Dose-response effect of 40 weeks of resistance training on bone mineral density in older adults. Osteoporosis International 201122 179-186. (doi:10.1007/s00198-0101182-9)

11 Marques EA, Wanderley F, Machado L, Sousa F, Viana JL, Moreira-Goncalves D, Moreira P, Mota J \& Carvalho J. Effects of resistance and aerobic exercise on physical function, bone mineral density, OPG and RANKL in older women. Experimental Gerontology 201146 524-532. (doi:10.1016/j.exger.2011.02.005)

12 Marques EA, Mota J \& Carvalho J. Exercise effects on bone mineral density in older adults: a meta-analysis of randomized controlled trials. Age 201234 1493-1515. (doi:10.1007/s11357011-9311-8)

13 Jarvinen TL, Sievanen H, Khan KM, Heinonen A \& Kannus P. Shifting the focus in fracture prevention from osteoporosis to falls. BMJ 2008 336 124-126. (doi:10.1136/bmj.39428.470752.AD)

14 Turner $\mathrm{CH}$. Three rules for bone adaptation to mechanical stimuli. Bone 199823 399-407. (doi:10.1016/S8756-3282(98)00118-5)

15 Clarke B. Normal bone anatomy and physiology. Clinical Journal of the American Society of Nephrology 2008 (Supplement 3) S131-S139. (doi:10.2215/CJN.04151206)

16 Tu X, Rhee Y, Condon KW, Bivi N, Allen MR, Dwyer D, Stolina M, Turner $\mathrm{CH}$, Robling AG, Plotkin LI et al. Sost downregulation and local Wnt signaling are required for the osteogenic response to mechanical loading. Bone 201250 209-217. (doi:10.1016/ j.bone.2011.10.025)

17 Clarke BL \& Drake MT. Clinical utility of serum sclerostin measurements. BoneKEy Reports 20132 361. (doi:10.1038/ bonekey.2013.95)

18 Polyzos SA, Anastasilakis AD, Bratengeier C, Woloszczuk W, Papatheodorou A \& Terpos E. Serum sclerostin levels positively correlate with lumbar spinal bone mineral density in postmenopausal women-the six-month effect of risedronate and teriparatide. Osteoporosis International 201223 1171-1176. (doi:10.1007/s00198010-1525-6)

19 Modder UI, Hoey KA, Amin S, McCready LK, Achenbach SJ, Riggs BL, Melton LJ 3rd \& Khosla S. Relation of age, gender, and bone mass to circulating sclerostin levels in women and men. Journal of Bone and Mineral Research 26 373-379. (doi:10.1002/jbmr.217)

20 He J, Zhang H, Wang C, Zhang Z, Yue H, Hu W, Gu J, Fu W, Hu Y, Li M et al. Associations of serum sclerostin and polymorphisms in the SOST gene with bone mineral density and markers of bone metabolism in postmenopausal Chinese women. Journal of Clinical Endocrinology and Metabolism 201499 E665-E673. (doi:10.1210/ jc.2013-2086)

21 Kanis JA, Oden A, Johansson H, Borgstrom F, Strom O \& McCloskey E. FRAX and its applications to clinical practice. Bone 200944 734-743. (doi:10.1016/j.bone.2009.01.373)
22 NIH Consensus Development Panel on Osteoporosis Prevention, Diagnosis, and Therapy. Osteoporosis prevention, diagnosis, and therapy. JAMA 2001285 785-795. (doi:10.1001/jama.285.6.785)

23 Adams JE. Quantitative computed tomography. European Journal of Radiology 200971 415-424. (doi:10.1016/j.ejrad.2009.04.074)

24 Cann CE, Adams JE, Brown JK \& Brett AD. CTXA hip-an extension of classical DXA measurements using quantitative CT. PLOS ONE 20149 e91904. (doi:10.1371/journal.pone.0091904)

25 Khoo BC, Brown K, Cann C, Zhu K, Henzell S, Low V, Gustafsson S, Price RI \& Prince RL. Comparison of QCT-derived and DXA-derived areal bone mineral density and $\mathrm{T}$ scores. Osteoporosis International 200920 1539-1545. (doi:10.1007/s00198-008-0820-y)

26 Eriksson JG, Sandboge S, Salonen MK, Kajantie E \& Osmond C. Long-term consequences of maternal overweight in pregnancy on offspring later health: findings from the Helsinki Birth Cohort Study. Annals of Medicine 201446 434-438. (doi:10.3109/07853890.2014. 919728)

27 Yliharsila H, Kajantie E, Osmond C, Forsen T, Barker DJ \& Eriksson JG. Birth size, adult body composition and muscle strength in later life. International Journal of Obesity 200731 1392-1399. (doi:10.1038/ sj.ijo.0803612)

28 Bucci M, Huovinen V, Guzzardi MA, Koskinen S, Raiko JR, Lipponen H, Ahsan S, Badeau RM, Honka MJ, Koffert J et al. Resistance training improves skeletal muscle insulin sensitivity in elderly offspring of overweight and obese mothers. Diabetologia 2015 59 77-86. (doi:10.1007/s00125-015-3780-8)

29 Epley B. Poundage chart. In Boyd Epley Workout. Lincoln, NE: Body Enterprises, 1985.

30 Santanasto AJ, Glynn NW, Newman MA, Taylor CA, Brooks MM, Goodpaster BH \& Newman AB. Impact of weight loss on physical function with changes in strength, muscle mass, and muscle fat infiltration in overweight to moderately obese older adults: a randomized clinical trial. Journal of Obesity 20112011 (doi:10.1155/2011/516576). Epub 2010 Oct 10.

31 Kakonen SM, Hellman J, Karp M, Laaksonen P, Obrant KJ, Vaananen HK, Lovgren T \& Pettersson K. Development and evaluation of three immunofluorometric assays that measure different forms of osteocalcin in serum. Clinical Chemistry 200046 332-337.

32 Lang TF, Saeed IH, Streeper T, Carballido-Gamio J, Harnish RJ, Frassetto LA, Lee SM, Sibonga JD, Keyak JH, Spiering BA et al. Spatial heterogeneity in the response of the proximal femur to two lower-body resistance exercise regimens. Journal of Bone and Mineral Research 201429 1337-1345. (doi:10.1002/jbmr.2155)

$33 \mathrm{Li} \mathrm{W}$, Sode M, Saeed I \& Lang T. Automated registration of hip and spine for longitudinal QCT studies: integration with 3D densitometric and structural analysis. Bone 200638 273-279. (doi:10.1016/ j.bone.2005.08.014)

34 Lang TF, Keyak JH, Heitz MW, Augat P, Lu Y, Mathur A \& Genant HK. Volumetric quantitative computed tomography of the proximal femur: precision and relation to bone strength. Bone 199721 101-108. (doi:10.1016/S8756-3282(97)00072-0)

35 Ma HT, Griffith JF, Xu L \& Leung PC. The functional muscle-bone unit in subjects of varying BMD. Osteoporosis International 201425 999-1004. (doi:10.1007/s00198-013-2482-7)

$36 \mathrm{Kaji} \mathrm{H}$. Linkage between muscle and bone: common catabolic signals resulting in osteoporosis and sarcopenia. Current Opinion in Clinical Nutrition and Metabolic Care 201316 272-277. (doi:10.1097/ MCO.0b013e32835fe6a5)

37 Jurimae J, Tillmann V, Cicchella A, Stefanelli C, Vosoberg K, Tamm AL \& Jurimae T. Increased sclerostin and preadipocyte factor-1 levels in prepubertal rhythmic gymnasts: associations with bone mineral density, body composition, and adipocytokine values. Osteoporosis International 201627 1239-1243. (doi:10.1007/ s00198-015-3301-0)

38 Shen H, Grimston S, Civitelli R \& Thomopoulos S. Deletion of connexin43 in osteoblasts/osteocytes leads to impaired muscle 
formation in mice. Journal of Bone and Mineral Research 201530 596-605. (doi:10.1002/jbmr.2389)

39 Falk B, Haddad F, Klentrou P, Ward W, Kish K, Mezil Y \& Radom-Aizik S. Differential sclerostin and parathyroid hormone response to exercise in boys and men. Osteoporosis International 2015 27 1245-1249. (doi:10.1007/s00198-015-3310-z)

40 Lombardi G, Lanteri P, Colombini A, Mariotti M \& Banfi G. Sclerostin concentrations in athletes: role of load and gender. Journal of Biological Regulators and Homeostatic Agents 201226 157-163.

41 Luo Q, Leng H, Wang X, Zhou Y \& Rong Q. The role of water and mineral-collagen interfacial bonding on microdamage progression in bone. Journal of Orthopaedic Research 201432 217-223. (doi:10.1002/ jor.22501)

42 Okano H, Mizunuma H, Soda M, Kagami I, Miyamoto S, Ohsawa M, Ibuki Y, Shiraki M, Suzuki T \& Shibata H. The long-term effect of menopause on postmenopausal bone loss in Japanese women: results from a prospective study. Journal of Bone and Mineral Research 199813 303-309. (doi:10.1359/jbmr.1998.13.2.303)

43 Cranney A, Horsley T, O'Donnell S, Weiler H, Puil L, Ooi D, Atkinson S, Ward L, Moher D, Hanley D et al. Effectiveness and safety of vitamin D in relation to bone health. Evidence Report/Technology Assessment 2007158 1-235.

44 Wamberg L, Pedersen SB, Richelsen B \& Rejnmark L. The effect of high-dose vitamin D supplementation on calciotropic hormones and bone mineral density in obese subjects with low levels of circulating 25-hydroxyvitamin d: results from a randomized controlled study. Calcified Tissue International 201393 69-77. (doi:10.1007/s00223-0139729-3)

45 Zhao J, Xia W, Nie M, Zheng X, Wang Q, Wang X, Wang W, Ning Z, Huang W, Jiang Y et al. The levels of bone turnover markers in Chinese postmenopausal women: Peking Vertebral Fracture study. Menopause 201118 1237-1243. (doi:10.1097/gme.0b013e31821d7ff7)

Received 18 June 2016

Revised version received 26 August 2016

Accepted 15 September 2016 